\section{Comparison of outcomes of free-hand 2-dimensional ultrasound-guided versus navigated 3-dimensional ultrasound- guided biopsy for supratentorial tumours: a single-institution experience with 125 cases}

Aditya D. Patil, Vikas Singh, Vivek Sukumar, Prakash M. Shetty, Aliasgar V. Moiyadi

Division of Neurosurgery, Department of Surgical Oncology, Tata Memorial Centre, Homi Bhabha National Institute, Mumbai, India

Purpose: The purpose of this study was to evaluate the relative utility and benefits of free-hand 2-dimensional intraoperative ultrasound (FUS) and navigated 3-dimensional intraoperative ultrasound (NUS) as ultrasound-guided biopsy (USGB) techniques for supratentorial lesions.

Methods: All patients who underwent USGB for suspected supratentorial tumours from January 2008 to December 2017 were retrospectively analyzed. The charts and electronic medical records of these patients were studied. Demographic, surgical, and pathological variables were collected and analyzed. The study group consisted of patients who underwent either FUS or NUS for biopsy.

Results: A total of 125 patients (112 adults and 13 children) underwent USGB during the study period (89 FUS and 36 NUS). NUS was used more often for deep-seated lesions ( $58 \%$ vs. 18\% for FUS, $\mathrm{P}<0.001$ ). The mean operating time for NUS was longer than for FUS (156 minutes vs. 124 minutes, $\mathrm{P}=0.001$ ). Representative yield was found in $97.7 \%$ of biopsies using FUS and in $100 \%$ of biopsies using NUS (diagnostic yield, $93.6 \%$ and $91.3 \%$, respectively). The majority of lesions (89\%) were high-grade gliomas or lymphomas. Postoperative complications were more common in the NUS group ( $8.3 \%$ vs. $1.2 \%$ ), but were related to the tumour location (deep).

Conclusion: Despite the longer operating time and higher rate of postoperative complications, NUS has the benefit of being suitable for biopsies of deep-seated supratentorial lesions, while FUS remains valuable for superficial lesions.

Keywords: Ultrasonography; Neuronavigation; Stereotactic; Biopsy; Glioma

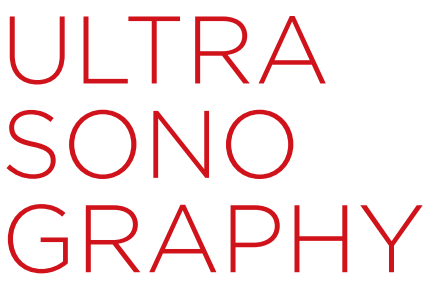

ORIGINAL ARTICLE

https://doi.org/10.14366/usg. 18036 pISSN: 2288-5919 • eISSN: 2288-5943 Ultrasonography 2019;38:255-263

Received: June 20, 2018

Revised: December 4, 2018

Accepted: December 8, 2018

Correspondence to:

Aliasgar V. Moiyadi, MCh, Division of Neurosurgery, Department of Surgical Oncology, Tata Memorial Centre, Homi Bhabha National Institute, Mumbai 400012, India

Tel. $+91-22-24177279$

Fax. +91-22-27405061

E-mail: aliasgar.moiyadi@gmail.com

This is an Open Access article distributed under the terms of the Creative Commons Attribution NonCommercial License (http://creativecommons.org/ licenses/by-nc/4.0/) which permits unrestricted noncommercial use, distribution, and reproduction in any medium, provided the original work is properly cited.

Copyright (C) 2019 Korean Society of Ultrasound in Medicine (KSUM)

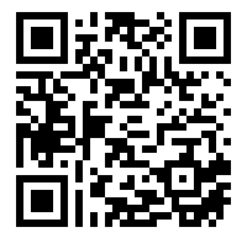

How to cite this article:

Patil AD, Singh V, Sukumar V, Shetty PM Moiyadi AV. Comparison of outcomes of free-hand 2-dimensional ultrasound-guided versus navigated 3-dimensional ultrasoundguided biopsy for supratentorial tumours: a single-institution experience with 125 cases. Ultrasonography. 2019 Jul;38(3):255-263. 


\section{Introduction}

Tissue diagnosis, through a biopsy or surgical resection, is essential for most intracranial masses, especially in the present molecular era in which adjuvant therapy is heavily guided by the correct pathological and molecular characterization of the tissue. Imageguided frame-based biopsy and frameless (neuronavigation) stereotactic biopsy are well-established techniques for achieving tissue diagnosis [1]. However, they are logistically challenging, cumbersome, and time-consuming in some circumstances due to the need for dedicated magnetic resonance imaging (MRI) before the procedure and coordinating patient flow between the operating room and radiology, particularly for frame-based techniques. Furthermore, they require patient co-operation (for frame-based techniques) and, most importantly, real-time monitoring is not possible intraoperatively. Intraoperative ultrasonography (US) is being increasingly used by neurosurgeons. It is a multimodal tool with many applications during surgery, of which one of the longestrecognized and most important is lesion localization. In addition, US can help in assessing the presence of residue, looking for hematomas, and identifying the surrounding normal anatomy, and it also provides information about the vasculature [2]. With advances in technology and the development of special small probes, it is possible to obtain accurate, high-resolution, and high-quality images of intracranial structures, even through burr holes or small craniotomies $[3,4]$. There is no need for preoperative navigation MRI sequences or fiducial markers, thus eliminating inaccuracies in image-to-patient registration. There are no brain shift issues, as the scans can be easily repeated as many times as needed and updated information is readily available, thereby providing truly "real-time" imaging $[2,5,6]$.

At our tertiary neuro-oncology centre, we have been using intraoperative US (IOUS) for over 10 years. Both conventional 2-dimensional (2D) US (2DUS) (since December 2007) and a navigated 3-dimensional (3D) US system (3DUS) (since June 2011) have been used. During this period, our surgical team performed USguided biopsies for various brain tumour-related indications. There were no specific policies regarding the use of any particular type of US technique. The decision was primarily based on the surgical team's preference depending on the case. We retrospectively reviewed our database to evaluate differences between these two groups.

\section{Materials and Methods}

We performed a retrospective analysis of all patients who underwent biopsy for intracranial lesions at our centre from January
2008 to December 2017. The retrospective analysis was approved by the institutional review board with a waiver of consent as per institutional policies. Patients who underwent stereotactic framebased or image-guided frameless biopsy (neuronavigation based on preoperative $M R I, n=11$ ) were excluded from the study. A total of 125 patients ( 81 males, 44 females; mean age, 46.6 years) who underwent intraoperative US-guided biopsy for supratentorial lesions were included in the study. All charts, files, and electronic medical records of these patients were studied. Demographic, surgical, and pathological variables were collected and analyzed.

The lesions were classified as deep or superficial, depending upon their location as seen on preoperative MRI. Deep tumours were defined as those whose epicentre was located in the thalamus, basal ganglia, internal capsule, corpus callosum, or deep white matter. Superficial lesions included those in the cortical or sub-cortical area. The senior author (A.V.M.) and a neurosurgical fellow (A.D.P.) jointly determined the classification of the tumour location as recorded in the charts.

The US-guided biopsy procedures were divided into two groups: $A$ and B. The 89 patients in group A underwent free-hand IOUS (FUS)-guided biopsy. In this group, after studying the MRI images in detail, an appropriate skin flap was marked and a small craniotomy (approximately $4 \mathrm{~cm} \times 4 \mathrm{~cm}$ ) was made under general anaesthesia. Standard 2DUS was used (Capasee II, Toshiba Corp., Tochigi Ken, Japan) with a linear 7-MHz transducer with a small foot plate (PVF738 F, Toshiba Corp.) and a Sonosite M-Turbo machine (Sonosite Inc., Bothell, WA, USA) with a variable-frequency (13-6 MHz) 25-mm-footprint broadband linear array transducer (L25X). In some cases, the Sonowand system (as a standalone 2DUS system without navigation) was used. The lesion was visualized using an appropriate probe. A small incision was made to open the dura and a freehand biopsy was performed, usually with biopsy cup forceps after performing a small corticectomy in some cases or using a Sedan side-cutting biopsy needle in others (no particular preference).

The 36 patients in group B underwent direct navigated 3D IOUS (NUS)-guided biopsy. In this group, the Sonowand system (SonoWand, SonoWand AS, Trondheim, Norway) was used for targeted biopsy. Although it can function as a routine navigation platform using preoperative MRI scans, it also has the capability of acquiring reformatted 3DUS images that can be tracked. The system can be used by itself as a stand-alone 2DUS system (as we did in some cases in group A) or as a direct NUS system $[6,7]$. A wide range of US probes are available, which facilitates intraoperative scanning and navigation, both superficially and deep in the parenchyma through small craniotomies. We generally used a flat phased array probe with a footprint of $25 \mathrm{~mm} \times 17 \mathrm{~mm}$ and a frequency range of 3-8 $\mathrm{MHz}$, which was suitable for both superficial 
and deep-seated targets at a depth varying from 1 to $12 \mathrm{~cm}$, with optimal imaging up to a depth of $8 \mathrm{~cm}$. For surfacing (cortical) lesions, a flat linear array probe $(32 \times 11$ - $\mathrm{mm}$ footprint, 6 - to 12 $\mathrm{MHz}$ range) was preferred. In this group, after performing a small craniotomy (no MRI-based navigation was used), real-time 3DUS acquisition of the tumour was done with a probe. The resulting data were registered in the system. Using a navigator, the exact target for the biopsy and the trajectory were planned. A small opening was made in the dura. A side-cutting needle mounted on the navigator was then passed along the trajectory planned under US-navigation guidance, using the target function to continuously track the tip of the biopsy needle, and multiple biopsies were taken (supplementary material with video of a navigated biopsy procedure) (Video clips 1 , 2).

After obtaining sufficient tissue samples for histopathology, real-time monitoring of the biopsy bed was done in both groups for at least 10 minutes to check for any hematoma formation in the biopsy bed (Fig. 1) and/or along the biopsy track (Fig. 2). The operating time was calculated from the records, which included the time for anaesthesia induction up to the completion of the surgical procedure. Postoperative imaging was done in all patients within 24 hours. Intraoperative frozen sections were sent in most cases (except when logistically not possible, outside of routine hours). We retrieved data from all pathology reports to ascertain the yield. The pathology reports were classified as diagnostic or positive (definite pathological diagnosis), representative but non-diagnostic (abnormal pathological tissue obtained, but no conclusive diagnosis could be reached), and negative (normal non-pathological tissue). We calculated the yield for diagnostic tissue (positive or conclusive pathological tissue), as well as all representative tissue (positive and representative reports).

The SPSS version 21.0 (IBM Corp., Armonk, NY, USA) was used for all statistical analyses. Only a descriptive analysis was done. Categorical variables were expressed as frequencies and percentages, and continuous variables as mean \pm standard deviation. Comparisons between groups for continuous variables were performed using the Student $t$ test, and the chi-square test was used to compare categorical variables. Statistical significance was ascertained by P-values of $<0.05$.

\section{Results}

A total of 125 biopsies in which IOUS was used were included in this study. Table 1 outlines the demographic and clinicopathological characteristics of the study group. The majority of the tumours were glial in origin $(55 \%)$, followed by lymphomas $(34 \%)$. The two cohorts were similar in most respects except for the location of the lesions, as group $B$ had significantly more deep lesions $(P<0.001)$. Furthermore, the operating time was longer in group $B$ by almost 30 minutes.

Diagnostic yield was calculated for the entire cohort. Intraoperative frozen sections were evaluated in 117 cases, and representative

Table 1. Demographic and clinicopathological characteristics of the cohort $(n=125)$

\begin{tabular}{lcc}
\hline & Group A (freehand US-guided) $(\mathrm{n}=89)$ & Group B (navigated 3D US-guided) ( $\mathrm{n}=36)$ \\
\hline Location & & P-value \\
Superficial & $13(82.0)$ & $15(41.7)$ \\
Deep & $16(18.0)$ & $21(58.3)$ \\
Sex & $58(65.2)$ & $23(63.9)$ \\
Male & $31(34.8)$ & $13(36.1)$ \\
Female & $49.0 \pm 17.9$ & $42.0 \pm 17.9$ \\
Age (yr) & $124.8 \pm 48.0$ & $157.2 \pm 54.0$ \\
Duration of surgery (min) & & $18(50.0)$ \\
Histological class & $49(55.1)$ & $12(33.3)$ \\
Glial tumors & $30(33.7)$ & $2(5.6)$ \\
Lymphomas & $3(3.4)$ & $1(2.8)$ \\
Others (demyelination, granulomas, etc.) & $1(1.1)$ & $3(8.3)$ \\
Embryonal tumors & $4(4.5)$ & 0.001 \\
Inconclusive & $2(2.2)$ & 0.001 \\
Negative & & 0.890 \\
\hline
\end{tabular}

Values are presented as number (\%) or mean \pm SD.

US, ultrasound; 3D, 3-dimensional; SD, standard deviation. 
tissue was confirmed (a positive diagnosis) in all cases. Final histology results were available in all 125 cases and were used to calculate the yield. The overall representative yield was $98.4 \%$. Representative tissue was obtained from 87 of 89 patients (97.75\%) in group A and 36 of 36 patients (100\%) in group B. Both patients with a negative yield had no frozen section sent. One patient underwent re-exploration and was diagnosed with anaplastic astrocytoma, while the other patient was lost to follow-up. There

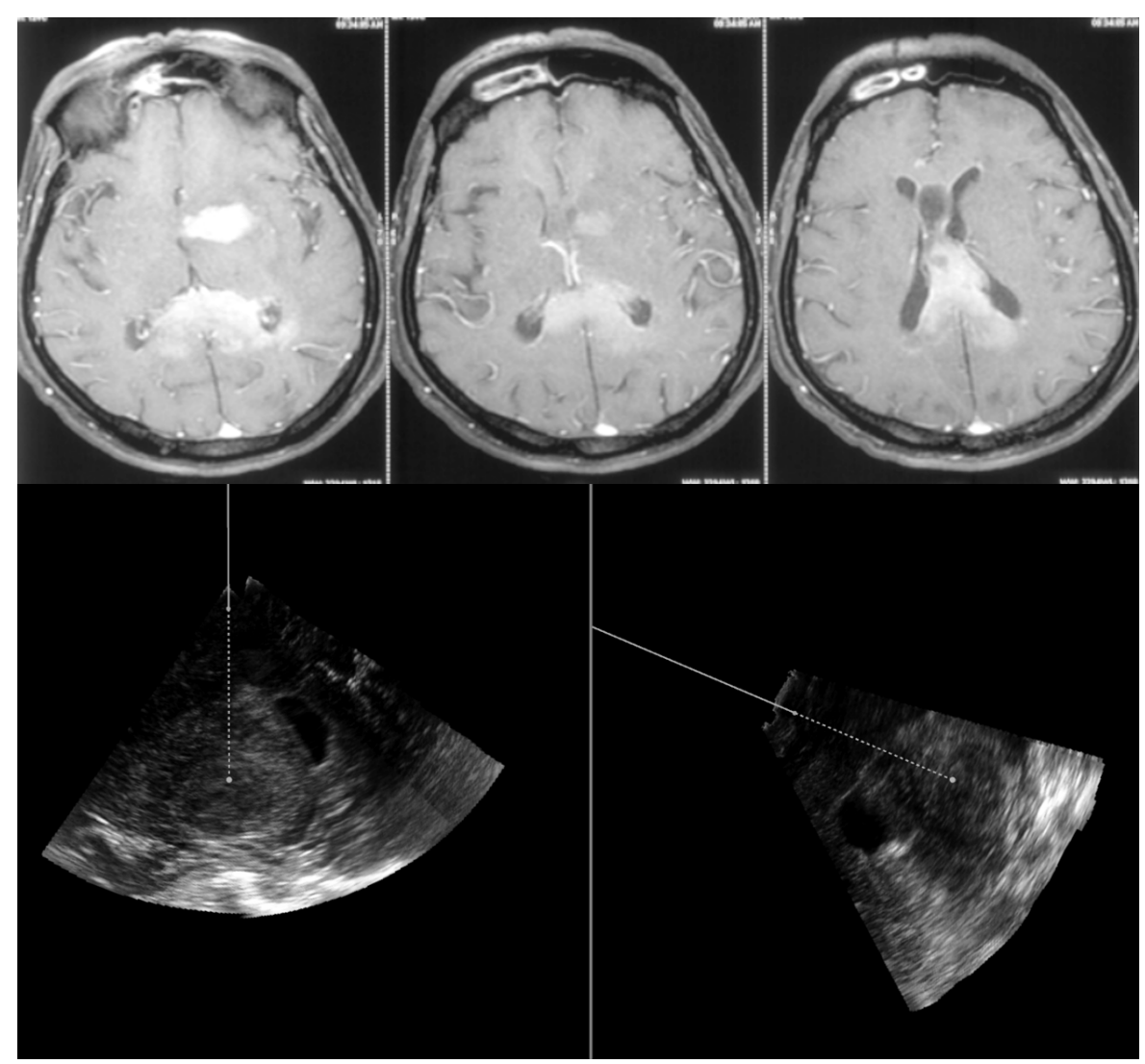

A

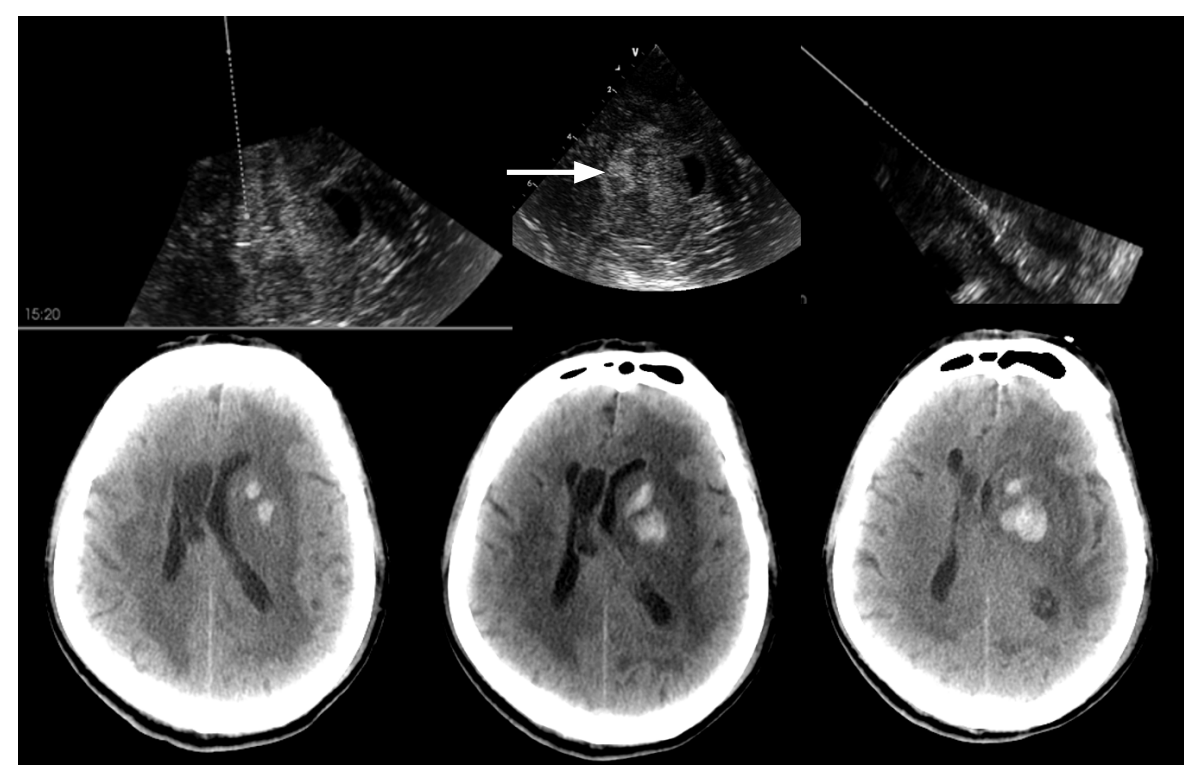

B
Fig. 1. Navigated 3D ultrasound-guided biopsy of a 70-year-old man with multiple intracranial lesions.

A. The upper panel shows axial T1-weighted magnetic resonance imaging with multiple homogenously enhancing lesions in the left gangliocapsular region, body, and splenium of the corpus callosum. The lower panel shows real-time intraoperative images obtained using 3-dimensional navigated ultrasound (two planes depicted side by side). The solid line represents the navigator, and the dotted lines represent the virtual offset planned for targeting the lesion for biopsy, along which the same navigatormounted needle is passed for biopsy. B. Intraoperative navigated ultrasound images (in three orthogonal planes) taken after biopsy of the lesion, show a small hematoma (arrow) in the tumour bed, along with track hematoma. Postoperative computed tomography images show a hematoma at the biopsy site (corresponding to the ultrasound images), which was managed conservatively. 


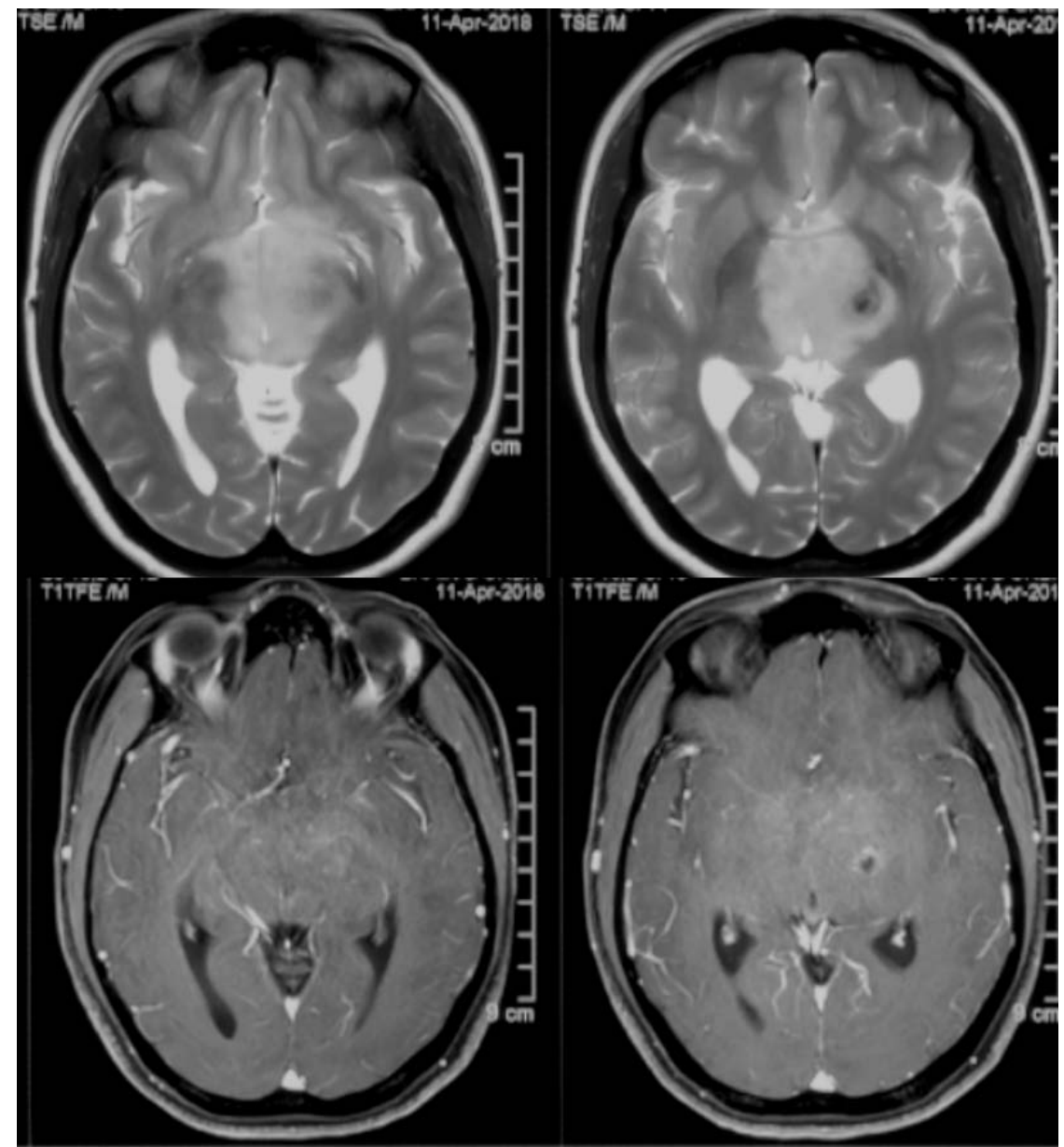

A

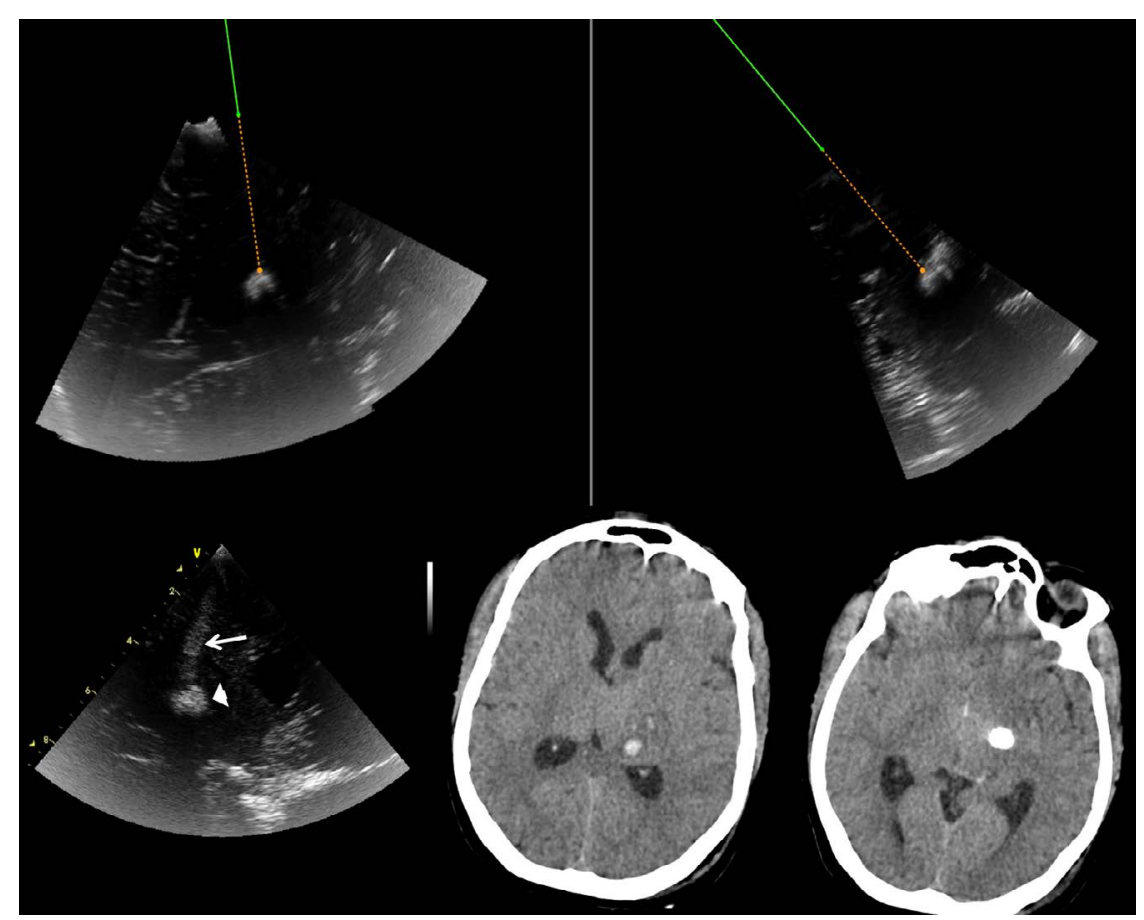

B
Fig. 2. Navigated 3D ultrasound-guided of a 35-year-old woman with bithalamic masses. A. Axial T2-weighted (top row) and postgadolinium contrast (bottom row) magnetic resonance images show diffuse and faintly enhancing lesions involving the bilateral thalami, with foci of suspected necrosis/ calcification in the left gangliocapsular lesion. B. Intraoperative ultrasound images of the same case are shown. The top row shows intraoperative ultrasound (navigated ultrasound) images in two planes, in which the target is set with an offset, and the virtual track along which the navigator-mounted needle is passed for biopsy. The lower post-biopsy ultrasound image (left) shows a small hematoma in the biopsy bed (arrowhead) and along the biopsy track (arrow) seen immediately after targeting the biopsy. Postoperative computed tomography scans show the small hematoma at the biopsy site (lower middle) and the deeper residual calcification (lower right). The hematoma was asymptomatic and conservatively managed. 
Table 2. Summary of complications in the study cohort

\begin{tabular}{lcccc}
\hline & $\begin{array}{c}\text { Neurological } \\
\text { worsening }(n=4)\end{array}$ & $\begin{array}{c}\text { Significant } \\
\text { hematoma }(n=3)\end{array}$ & $\begin{array}{c}\text { Perioperative } \\
\text { mortality }(n=3)\end{array}$ & $\begin{array}{c}\text { Re-exploration for } \\
\text { negative yield }(n=4)\end{array}$ \\
\hline $\begin{array}{l}\text { Free-hand US-guided }(n=89) \\
\text { Superficial }\end{array}$ & 0 & 0 & 0 & 1 \\
Deep-seated & 1 & 1 & 1 & 0 \\
Navigated US-guided ( $n=36)$ & 1 & 0 & 1 & 0 \\
Superficial & 2 & 2 & 1 & 0 \\
Deep-seated & & & & 0 \\
\hline
\end{tabular}

US, ultrasound.

were seven cases (4 in group A, 3 in group B) with inconclusive histopathology reports, with findings of reactive gliosis, nonspecific inflammatory reaction, and extensive necrosis. Therefore, the diagnostic (positive) yield was $92.8 \%$ overall (group A, 93.6\%; group $B, 91.3 \% ; P>0.05)$.

The complications are shown in Table 2. Significant hematoma formation occurred in one patient in group A with a corpus callosal glioma. This patient was re-explored for hematoma evacuation and decompression, but progressively worsened and died. One patient in group B developed intraventricular hemorrhage following biopsy of a thalamic glioma and required an external ventricular drain insertion, but recovered well. Two post-procedural deaths occurred in group B (5.55\%): one due to an intratumoural bleed in a glioblastoma multiforme patient and other due to progressive worsening in a non-Hodgkin lymphoma patient. All hematomas that occurred postbiopsy in either group, as shown in Table 2, were in patients with deep-seated lesions. Three of the 36 patients in group B experienced neurological worsening, and two of those patients had deep-seated tumours. This indicates that neurological worsening and hematoma development were associated with the location of the tumour, rather than the US modality used for biopsy. Minor complications such as superficial wound-related complications occurred in five of the 89 patients $(5.61 \%)$ in group $A$, and one of the 36 patients $(2.77 \%)$ in group $B$. No patient developed meningitis.

\section{Discussion}

IOUS has been increasingly utilized as a tool for surgical resections and biopsies in the last 3 decades due to its advantage of real-time monitoring. IOUS has been utilized as a multimodal tool in tumour surgery for localizing tumours intraoperatively, as a tool for resection control, and for biopsy of intracranial lesions [8,9]. Meanwhile, navigation technology has seen exponential development and improvements, and many options for targeted biopsy are now available. A recent large series by Lu et al. [10] compared frame- based, frameless, and intraoperative MRI-guided intracranial biopsies. Interestingly, they found the best accuracy with framebased biopsies (96\%) and the lowest with intraoperative MRIguided biopsies (89\%). IOUS is a useful cost-effective alternative to intraoperative MRI. Multiple studies have compared IOUS with the standard frame-based and frameless techniques [11-13], and all have highlighted the benefits of US, especially its real-time control.

US-guided biopsies have generally been performed using 2DUS, either free-hand or with various kinds of biopsy guides affixed to the probes $[5,13-16]$. NUS is a new modality that has evolved in the last decade $[2,17]$. In the current study, we present our experiences with a series of 125 consecutive brain lesion biopsies using two different IOUS techniques. Based on an extensive search of the literature, to the best of our knowledge, no study has yet compared free-hand 2DUS-guided and navigated 3DUS-guided biopsies. In both groups, the diagnostic yield was comparable (FUS, 93.26\%; NUS, 91.67\%; overall, 92.8\%), and in accordance with previous series of IOUS-guided biopsies in the literature $[5,11,12,14]$. Allouch et al. [5] reported one of the largest series of US-guided biopsies. They used 2DUS coupled with a biopsy guide and superimposed the trajectory on the sonograms for guidance. In their series, only $16 \%$ of the tumours were deep. They achieved a diagnostic yield of $92 \%$, with representative tissue obtained in $95 \%$ of the 100 cases [5]. Satyarthee et al. [11] reported a similar yield of $91 \%$ in their study of 22 IOUS-guided biopsies. Occasionally, even though the target is correctly reached and the tissue obtained is abnormal, a definitive pathological diagnosis is not possible. Such lesions are generally labelled as representative but non-diagnostic (or inconclusive). Non-enhancing lesions are more prone to non-diagnostic yield [5]. Although it is possible that the target may have been missed, in such cases, the pathological examination would usually return a negative report, not an indeterminate one. This is actually a limitation of the histological evaluation, rather than of the technique of targeting the lesion. Considering all such non-diagnostic but representative samples together, our representative yield was $98.4 \%$ overall. 
We managed to obtain a representative tissue sample in all 36 of 36 NUS cases. This could have been due the advantage of better accuracy, as well as the ability to confirm that the biopsy needle was positioned within the target in real-time, which is not possible with stereotactic and other conventional navigated techniques. IOUS also has the advantage of allowing the surgeon to account for the brain shift that can occur after opening the dura, or due to the collapse of tumour cysts. In our study, both the negative biopsies were in the FUS group. There were no negative biopsies in the NUS group, underscoring the superior accuracy of this technique.

The mean operating time in the current study for the FUS group was 124.8 minutes, as compared to 157.2 minutes for the NUS group. This included the entire duration of the operating room time, including the preoperative anaesthesia time and postoperative procedures. The NUS group required approximately 30 minutes more. This may have been due to the time required for 3D acquisition of the tumour by IOUS and saving the data in the system, as well as the early phase of the surgeon's learning curve with the system. Allouch et al. reported a mean or time of 45 minutes from skin incision to closure [5]. Satyarthee et al. [11] and Di Lorenzo et al. [12] reported mean times of 94 minutes and 56 minutes, respectively, but did not define operating time. All our patients underwent biopsy under general anaesthesia followed by a small craniotomy $(3-4 \mathrm{~cm})$ for biopsy. Even though smaller burr hole probes are available, in our experience, the information provided by a larger (2-cm footprint) probe outweighs the perceived drawbacks of a larger bone flap. The mean operating room time required in our study is comparable to that reported in the literature for frame-based and frameless stereotactic biopsies (114 and 185 minutes, respectively) [18-20].

Significant neurological worsening due to hematoma formation occurred in $1.12 \%$ of patients ( 1 of 89 ) in the FUS group and in $8.33 \%$ of patients ( 3 of 36 ) in the NUS group. This can be explained by selection bias. Of the 36 patients who underwent NUS-guided biopsy, $21(58.33 \%)$ had deep lesions, in contrast to 16 of the 89 patients $(17.97 \%)$ who underwent FUS-guided biopsy $(P<0.001)$. For deep-seated "difficult" lesions, it is likely that the operating surgeon preferred to use NUS due to its theoretical advantages. Again, the results were in accordance to those reported in the literature, with significant hematoma formation in $4 \%-5 \%$ of cases and neurological worsening in $5 \%-13 \%$ of cases $[5,11,12]$. Using the duplex mode of US for delineation of the vessels, a safe trajectory can be determined for biopsy. As reported by Allouch et al. $[5,21]$, this does not necessarily translate into a lower rate of complications, which probably occur due to the abnormal and fragile microvasculature. However, IOUS helps detect complications on the operating table, allowing clinicians to anticipate possible postoperative problems and to institute appropriate measures as soon as possible $[5,21]$. Of the three patients with significant hematoma in the NUS group, one required evacuation of an intratumoural hematoma, one required external ventricular drain insertion for an intraventricular bleed, and one experienced progressive worsening and death due to an intratumoural bleed. One patient in the FUS group was re-explored for intratumoural hematoma formation and mass effect.

The main limitation of this study is its non-randomized and retrospective nature. The selection of the US modality used for the lesion depended upon the surgeon's preference and comfort level. Our series contained an overrepresentation of deep-seated lesions in the NUS group. It is possible that the operating surgeon chose to biopsy more "difficult" cases using NUS due to its theoretical advantages, whereas more superficial lesions were targeted with FUS. Nonetheless, this is a large series of US-guided biopsies using two different techniques that provides insights into the advantages and disadvantages of each.

Nevertheless, NUS has major benefits for biopsies of intracerebral lesions. Frame-based techniques have long been considered the gold standard for targeting intracranial lesions, but they are logistically challenging, due to factors including the bulkiness of frames, the patient's discomfort, shifting the patient to computed tomography or MRI with the frame, and the calculations required for planning the trajectory $[20,22-24]$. Direct NUS does not interfere with the surgical workflow. It eliminates the need for obtaining navigation sequences of preoperative MRI. This saves time and money. There is no need for any special instruments, except for a calibrated US apparatus, and no need for radiologists or technicians. It adds very little extra time to the operation, and the investment costs are considerably lower than for intraoperative MRI. It eliminates the inaccuracies inherent to image-to-patient registration algorithms, because the acquisition and display of the images are in the same reference frame. The scans are very easy and convenient to obtain, requiring no more than a few minutes each time an update is needed. Therefore, scans can be repeated as many times as needed [6]. Interpretation of the sonograms (in the absence of a full head view) can be challenging for less-experienced users. However, by insonating a large area to include the surrounding brain and known anatomical landmarks, not just the tumour area, a larger $3 \mathrm{D}$ volume can be reconstructed, and this considerably improves the orientation. Image quality may sometimes be suboptimal. A meticulous technique during insonation (easily mastered with experience over time) and avoidance of artefacts can minimize most causes of suboptimal image quality $[2,25]$.

In conclusion, IOUS-guided biopsy is a safe, simple, and technically reliable real-time method for biopsies of both superficial and, in particular, deep-seated intracerebral lesions. Compared 
to other real-time intraoperative imaging, direct navigated 3DUS technology is advantageous in many ways. It provides an equally effective alternative to frame-based or frameless stereotactic biopsy, with the added benefit of real-time monitoring of post-procedure intratumoural bleeds. However, even free-hand 2DUS guidance is a valuable tool for superficial lesions. Experience with sonogram acquisition and interpretation is essential to extract the maximum benefit from this potentially useful adjunct.

ORCID: Aditya D. Patil: https://orcid.org/0000-0001-9057-7724; Aliasgar V. Moiyadi: https://orcid.org/0000-0002-4082-2004

\section{Author Contributions}

Conceptualization: Moiyadi AV. Data acquisition: Patil AD, Singh V, Sukumar V. Data analysis or interpretation: Patil AD, Singh V, Sukumar V. Drafting of the manuscript: Patil AD, Singh V. Critical revision of the manuscript: Shetty PM, Moiyadi AV. Approval of the final version of the manuscript: all authors.

\section{Conflict of Interest}

No potential conflict of interest relevant to this article was reported.

\section{Supplementary Material}

Video clip 1. Setting up of biopsy using navigated 3-dimensional ultrasound. In this case a biopsy from the edge of the tumor is being planned as part of another parallel study (case not part of the present paper). The ultrasound has already been acquired and has been overlaid with the magnetic resonance. The intended biopsy point is selected on the screen (as a blue spot). The solid green line corresponds to the navigator position and the orange dotted line is the extended projection of the navigator to select the biopsy point. As soon as the biopsy point is marked the system switches to a dual plane view (one plane parallel to the intended trajectory, and the other perpendicular to it in an end-on plane). Once the biopsy point is fixed, the navigated biopsy needle (not shown in this video) is passed along the preselected trajectory. As the needle is advanced and as long as the trajectory is accurate the biopsy point is surrounded by a green circle. Any deviation from the trajectory shows up as a red circle warning the surgeon. Once the biopsy point is reached, the biopsy point is surrounded by a light blue circle indicating successful targeting of the marked point (https://doi. org/10.14366/usg.18036.v001).

Video clip 2. Navigated biopsy of a thalamic lesion. In this case only a direct 3-dimensional ultrasound has been used for navigation. No preoperative magnetic resonance imaging was used (https://doi. org/10.14366/usg.18036.v002).

\section{References}

1. Apuzzo ML, Chandrasoma PT, Cohen D, Zee CS, Zelman V. Computed imaging stereotaxy: experience and perspective related to 500 procedures applied to brain masses. Neurosurgery 1987;20:930-937.

2. Moiyadi AV. Intraoperative ultrasound technology in neuro-oncology practice: current role and future applications. World Neurosurg 2016;93:81-93.

3. Moringlane JR, Voges M. Real-time ultrasound imaging of cerebral lesions during "target point" stereotactic procedures through a burr hole. Technical note. Acta Neurochir (Wien) 1995;132:134137.

4. Walter U, Kanowski M, Kaufmann J, Grossmann A, Benecke R, Niehaus L. Contemporary ultrasound systems allow high-resolution transcranial imaging of small echogenic deep intracranial structures similarly as MRI: a phantom study. Neuroimage 2008;40:551-558.

5. Allouch $H$, Pfeifenbring S, Behnke-Mursch J, Halatsch ME, Mursch K. Real-time ultrasound monitoring during intracranial needle biopsies: operative results and detection of complications in 100 cases. World Neurosurg 2014;82:202-206.

6. Moiyadi AV, Shetty P. Direct navigated 3D ultrasound for resection of brain tumors: a useful tool for intraoperative image guidance. Neurosurg Focus 2016;40:E5.

7. Moiyadi AV, Shetty P, Degaonkar A. Resection of pediatric brain tumors: intraoperative ultrasound revisited. J Pediatr Neurosci 2017;12:19-23.

8. Knake JE, Chandler WF, Gabrielsen TO, Latack JT, Gebarski SS. Intraoperative sonography in the nonstereotaxic biopsy and aspiration of subcortical brain lesions. AJNR Am J Neuroradiol 1983;4:672-674.

9. Sutcliffe JC, Battersby RD. Intraoperative ultrasound-guided biopsy of intracranial lesions: comparison with freehand biopsy. $\mathrm{Br} J$ Neurosurg 1991;5:163-168.

10. Lu Y, Yeung C, Radmanesh A, Wiemann R, Black PM, Golby AJ. Comparative effectiveness of frame-based, frameless, and intraoperative magnetic resonance imaging-guided brain biopsy techniques. World Neurosurg 2015;83:261-268.

11. Satyarthee GD, Chandra PS, Sharma BS, Mehta VS. Comparison of stereotactic and ultrasound-guided biopsy of solid supratentorial tumor: a preliminary report. Asian J Neurosurg 2017;12:664-669.

12. Di Lorenzo N, Esposito V, Lunardi P, Delfini R, Fortuna A, Cantore $G$. A comparison of computerized tomography-guided stereotactic and ultrasound-guided techniques for brain biopsy. J Neurosurg 1991;75:763-765.

13. Jain D, Sharma MC, Sarkar C, Gupta D, Singh M, Mahapatra AK. Comparative analysis of diagnostic accuracy of different brain biopsy procedures. Neurol India 2006;54:394-398.

14. Lunardi P, Acqui M, Maleci A, Di Lorenzo N, Fortuna A. Ultrasound- 
guided brain biopsy: a personal experience with emphasis on its indication. Surg Neurol 1993;39:148-151.

15. Berger MS. Ultrasound-guided stereotaxic biopsy using a new apparatus. J Neurosurg 1986;65:550-554.

16. Benediktsson $H$, Andersson $T$, Sjolander $U$, Hartman $M$, Lindgren PG. Ultrasound guided needle biopsy of brain tumors using an automatic sampling instrument. Acta Radiol 1992;33:512-517.

17. Unsgaard G, Rygh OM, Selbekk T, Muller TB, Kolstad F, Lindseth F, et al. Intra-operative 3D ultrasound in neurosurgery. Acta Neurochir (Wien) 2006;148:235-253.

18. Smith JS, Quinones-Hinojosa A, Barbaro NM, McDermott MW. Frame-based stereotactic biopsy remains an important diagnostic tool with distinct advantages over frameless stereotactic biopsy. J Neurooncol 2005;73:173-179.

19. Rajshekhar V. Current status of stereotactic biopsy. Stereotact Funct Neurosurg 2001;76:137-139.

20. Sawin PD, Hitchon PW, Follett KA, Torner JC. Computed imagingassisted stereotactic brain biopsy: a risk analysis of 225 consecutive cases. Surg Neurol 1998:49:640-649.
21. Allouch H, Behnke-Mursch J, Mursch K. Intra-operative diagnosis and image-guided management of an intracerebral haemorrhage occurring during ultrasound-guided biopsy. Acta Neurochir (Wien) 2007;149:91-93.

22. Woodworth GF, McGirt MJ, Samdani A, Garonzik I, Olivi A, Weingart JD. Frameless image-guided stereotactic brain biopsy procedure: diagnostic yield, surgical morbidity, and comparison with the framebased technique. J Neurosurg 2006;104:233-237.

23. Dammers R, Haitsma IK, Schouten JW, Kros JM, Avezaat CJ, Vincent AJ. Safety and efficacy of frameless and frame-based intracranial biopsy techniques. Acta Neurochir (Wien) 2008;150:23-29.

24. Sumanaweera TS, Adler JR Jr, Napel S, Glover GH. Characterization of spatial distortion in magnetic resonance imaging and its implications for stereotactic surgery. Neurosurgery 1994;35:696703.

25. Unsgard G, Solheim O, Lindseth F, Selbekk T. Intra-operative imaging with 3D ultrasound in neurosurgery. Acta Neurochir Suppl 2011;109:181-186. 